\title{
ORIGINAL ARTICLE Effects of chronic pain on quality of life and depression in patients with spinal cord injury
}

\author{
E Ataoğlu, T Tiftik, M Kara, H Tunç, M Ersöz and S Akkuş
}

Design: A cross-sectional study.

Objective: To assess the effects of pain on quality of life (QoL), functional independence and depression in patients with spinal cord injury (SCI).

Setting: An inpatient rehabilitation center.

Methods: A total of 140 patients $(104 \mathrm{M}, 36 \mathrm{~F})$ with $\mathrm{SCl}$ who underwent inpatient rehabilitation treatment were examined. A questionnaire including clinical variables was applied. Motor score of Functional Independence Measure was used to assess dailylife activities, the 36-Item Medical Outcomes Short-Form Health (SF-36) for QoL and Beck Depression Inventory (BDI) for depression. Patients were then divided into those having chronic pain (Group I) and those without any pain (Group II), and groups were compared according to demographic and clinical variables.

Results: The most common causes of SCl were falls (35.0\%) and motor vehicle accidents (34.2\%). Chronic pain was present in $78 \%$ of patients. Patients employed before injury and patients who had complete injury had lower Numerical Rating Scale scores $(P<0.05)$. SCl patients with chronic pain had higher depression ratings and their BDI scores were correlated with some of the SF-36 domains (general health, vitality, social functioning and mental health). Only bodily pain and social functioning $(P<0.05)$ scores were found to be lower in Group I $(P<0.05)$ when compared with Group II.

Conclusion: As mood and QoL are negatively affected with pain in $\mathrm{SCl}$ patients, we suggest that chronic pain should always be treated in a multidisciplinary setting where pharmacological, physical and psychological therapies are combined.

Spinal Cord (2013) 51, 23-26; doi:10.1038/sc.2012.51; published online 1 May 2012

Keywords: spinal cord injury; pain; quality of life; depression

\section{INTRODUCTION}

Spinal cord injury (SCI) causes many health-related problems affecting not only the patient's physical state but also all aspects of their lives, such as mood and health-related quality of life (QoL). Several studies using the 36-Item Medical Outcomes Short-Form Health Survey (SF-36) showed that QoL (nearly all of the SF-36 subscales) was low in patients with SCI. ${ }^{1,2}$ SF-36 is a reliable measure of QoL and has been commonly used to assess the disease impact. ${ }^{1}$ As SCI victims are generally active young adults and their disabilities last for the rest of their lives, studying QoL in this population is important.

Chronic pain is a common and significant complication in SCI patients. The prevalence of pain after SCI has been reported as $11-94 \%$ of the patients. ${ }^{3}$ Although the pain often starts within the initial 6 months of SCI, it frequently persists and sometimes can even become aggravated over time. ${ }^{4}$ As pain is known to affect recreational activities and vocational status, ${ }^{5}$ quality of sleep ${ }^{6}$ and sexuality, it can have a major role in QoL, mood and rehabilitation outcome. For many of them, the pain is severe and accompanied by reduced physical functioning, eventually lowering the QoL. ${ }^{8}$ Further, chronic pain and psychological disorders are closely related, affecting physical and psychosocial functioning. Likewise, studies show that an important proportion of SCI patients are depressed when compared with healthy subjects. ${ }^{9,10}$ It has also been shown that chronic pain is associated with worse depressive symptoms in these patients. ${ }^{11}$ Moreover, factors such as spasticity, socioeconomic disadvantage and medical complications have been found to be associated with lower QoL as well. ${ }^{1}$

Although chronic pain can be expected to have a negative impact on QoL and mood in SCI patients, to our best notice, no individual study has been performed in this regard. Accordingly, the purpose of this current study was twofold; first, we aimed to assess the effects of pain on QoL and mood in SCI patients; second, we aimed to find out the prevalence and characteristics of different types of SCI-related chronic pain and to explore whether there was any additional relationship between chronic pain and clinical characteristics of the patients.

\section{MATERIALS AND METHODS}

A total of 140 patients $(104 \mathrm{M}, 36 \mathrm{~F})$ with SCI who underwent inpatient rehabilitation treatment at Ankara Physical Medicine and Rehabilitation Education and Research Hospital were examined by the same physiatrist. This hospital is one of the largest rehabilitation centers where patients are admitted from all over our country. Patients were usually in the subacute or chronic stage, clinically stable, and they had already received necessary surgical interventions at the acute stage. Subjects included in the study were aged 
older than 16 years, able to speak and understand the tests, and had SCI with a duration of at least 3 months. Subjects were excluded from the study if they had concomitant traumatic brain injury, cognitive dysfunction and pain less than 3 months. All subjects were informed about the study procedure and they gave written informed consent. The study protocol was approved by the local ethics committee.

A questionnaire gathering information for age, sex, employment status before injury, level of education, date of injury, type, onset and duration of pain, pain intensity and prevalence, and level of injury was applied to all patients. Severity of injury was based on the ASIA impairment scale as ASIA A (no motor or sensory function in the sacral segments S4-S5) through ASIA E (normal motor and sensory function). Level of injury was classified as follows: C1-C8 as cervical injury; T1-T12 as thoracic injury and L1-S4/5 as lumbosacral injury. Patients were grouped according to the level of injury and general pain location (in areas of the body above injury level, below injury level or at injury level). Motor score of Functional Independence Measure (FIM) was used for daily-life activities; SF-36 for QoL and Beck Depression Inventory $(\mathrm{BDI})^{12}$ for depression were also used. BDI is a commonly used measurement to evaluate the depressed mood in chronic pain samples, such as SCI. It consists of 21 items rated on a categorical scale. We also graded these scores for depression severity according to Kendall et al., ${ }^{13}$ as $0-9$ normal, 10-19 mild depression, 20-30 moderate depression and 31-63 severe depression

Patients were then divided into two groups, as those complaining of chronic pain (lasting more than 3 months) (Group I) and those without pain (Group II), and pain scales were used only in Group I. Pain location was assessed by using McGill Pain Questionnaire. Patients were asked to rate their average pain intensity during the past week on a 0-10 Numerical Rating Scale (NRS), with $0=$ 'no pain' and $10=$ 'pain as bad as could be. ${ }^{14}$ NRS has been shown to have a good test-retest reliability and adequate validity in terms of associations with other pain measures and treatments. ${ }^{15}$ The Turkish version of Leeds Assessment of Neuropathic Symptoms and Signs (LANSS) scale was used to discriminate between neuropathic and non-neuropathic pain. ${ }^{16}$ It evaluates five types of pain: (a) thermal, (b) dysesthesia, (c) paroxysmal, (d) evoked and (e) autonomic dysfunction. Scores of 12 or higher (out of a total of 24) were considered neuropathic pain. Pain type was classified into four categories according to location, description and apparent origin as described elsewhere. ${ }^{14}$

\section{Statistical analysis}

SPSS (Statistical Package for Social Sciences, SPSS Inc, Chicago, IL, USA) version 15.0 was used for statistical analyses. Comparisons between the groups were performed with chi-square test, Mann-Whitney $U$-test and KruskalWallis test (for non-normal distributed variables), and Student's $t$-test (for normal distributed variables) where available. Spearman's rank coefficients were used for correlation analysis. A $P$ value of less than 0.05 was considered to be significant.

\section{RESULTS}

Socio-demographic and clinical characteristics of the patients are shown in Table 1. The most common causes of SCI were falls $(35.0 \%)$ and motor vehicle accidents $(34.2 \%)$. The two groups were similar with regard to clinical and demographic data. According to the cause of injury, only the frequency of SCI due to falls was significantly higher in Group I (39.4\%) when compared with Group II $(19.3 \%)$ $(P<0.05)$. Pain was more common in patients with SCI, resulting from falls $(87.8 \%)$ than from other causes.

In $109(77.8 \%)$ patients, chronic pain was present. Symptoms started within the initial 6 months after injury in $93(85.3 \%)$ patients with SCI. We found no significant relationship between pain intensity and age, gender, duration/level of injury and education. However, patients who were employed before SCI $\left(\chi^{2}=11.67, P<0.05\right)$ and patients who had complete injury $(P<0.05)$ had lower NRS scores (Table 2). Further, pain intensity correlated positively with the
Table 1 Study sample characteristics $(n=140)$

Variable

Age (years), mean \pm s.d.

$36.2 \pm 13.5$

Duration since injury (months), mean \pm s.d.

$25.2 \pm 43.9$

Gender, n (\%)

Male

$104(74.3)$

Female

$36(25.7)$

Education, $n$ (\%)

Illiterate

11 (7.9)

Some high school or lower

$90(64.2)$

High school degree

$28(20.0)$

College graduate or higher

11 (7.9)

Employment before injury, n (\%)

Employed

67 (47.9)

Retired

$13(9.3)$

Homemaker

27 (19.3)

Unemployed

$33(23.6)$

Cause of SCI, n (\%)

Fall

49 (35.0)

Motor vehicle accident

$48(34.2)$

Weight falling over

6 (4.3)

Gunshot wound

$8(5.7)$

Nontraumatic

29 (20.8)

Level of injury, n (\%)

C1-C8

$30(21.4)$

$\mathrm{T} 1-\mathrm{T} 12$

$77(55.0)$

$\mathrm{L} 1-\mathrm{S} 4 / 5$

$33(23.6)$

Completeness of injury, $n$ (\%)

Complete (ASIA A)

$79(56.4)$

Incomplete (ASIA B-E)

$61(43.6)$

Abbreviation: $\mathrm{SCl}$, spinal cord injury.

alatrogenic, vascular, neoplasia, infection.

Table 2 Pain intensity according to the completeness of injury and employment status before injury in Group I (mean \pm s.d. (min-max))

\begin{tabular}{lc}
\hline Variable & NRS score \\
\hline Employment & \\
$\quad$ Unemployed $(n=22)$ & $4.72 \pm 1.20(2-7)$ \\
Employed $(n=58)^{*}$ & $4.36 \pm 0.93(2-6)$ \\
Homemaker $(n=21)$ & $5.05 \pm 1.28(3-8)$ \\
Retired $(n=8)$ & $5.00 \pm 1.07(4-7)$ \\
Completeness of injury** & \\
Complete $(n=63)$ & $4.44 \pm 1.10(2-7)$ \\
Incomplete $(n=46)$ & $4.84 \pm 1.05(3-8)$
\end{tabular}

Abbreviation: NRS, Numerical Rating Scale.

*Kruskal-wallis test, $\chi^{2}=7.821, P<0.05$.

**Student's $t$-test, $P<0.05$

duration of injury $(r=0.266, P<0.05)$. Patients were more likely to report pain below the level of injury. Thoracic injury was present in $57.6 \%$ (60 patients) of those patients reporting pain below the level of injury. Patients rated their legs $(62.1 \%)$ and low back $(20.0 \%)$ as the most painful sites.

According to the clinical evaluation, neuropathic pain was the most common type ( 80 patients, $73.4 \%$ ). All of these 80 patients had belowlevel neuropathic pain and $11(13.8 \%)$ patients had at-level neuropathic pain as well. Musculoskeletal pain was the second most common type of pain experienced (in 58 patients, 53.2\%) and 
visceral pain was present only in $3(2.8 \%)$ patients. According to LANSS, neuropathic pain was present in $66(60.6 \%)$ patients. Among all painful patients, 72 patients $(66.1 \%)$ reported medication use for pain treatment.

According to the FIM motor scores, there was no significant difference between Group I (35.2 \pm 15.3$)$ and Group II (35.4 \pm 16.0$)$. Although mean BDI scores were found to be higher in Group I $(22.1 \pm 11.7)$ when compared with Group II $(17.1 \pm 12.2)(P=0.021)$, these scores did not correlate with NRS and FIM motor scores. On the other hand, BDI scores were negatively correlated with some of the SF-36 domains $(r=-0.229, P=0.016$ for general health, $r=-0.329, P=0.000$ for vitality, $r=-0.283, P=0.003$ for social functioning and $r=-0.247, P=0.010$ for mental health) in Group I. According to the depression severity, only moderate depression was found to be significantly higher in Group I (30.2\% of patients) than in Group II ( $6.4 \%$ of patients) $\left(\chi^{2}=7.960, P<0.05\right.$; Figure 1$)$.

Table 3 compares the scores of all SF-36 domains of both groups. The poorest SF-36 subscale scores were in physical functioning, rolephysical, role-emotional and general health in both groups (Table 3$)$. Only bodily pain $(P<0.001)$ and social functioning $(P=0.047)$ scores were found to be lower in Group I when compared with Group II. In Group I, NRS were correlated with only physical functioning $(r=0.201, P=0.039)$ and bodily pain $(r=-0.237$, $P=0.013)$. Factors like age, sex, time since injury, level and severity of injury (complete vs incomplete) have not been found in association with lower QOL domains in Group I.

\section{DISCUSSION}

This study demonstrated that pain after SCI is a common problem with a prevalence of $77.9 \%$. The most common causes of the SCI were falls $(35.0 \%)$ and motor vehicle accidents (34.2\%). Neuropathic (73.4\%) and musculoskeletal pain (53.2\%) were the most common types of painful conditions in these patients. Patients who had been working before the injury and patients with complete injury had lower NRS scores. SCI patients with chronic pain had higher depression ratings and their BDI scores were negatively correlated with some of the SF-36 domains (general health, vitality, social functioning and mental health). Only bodily pain and social functioning scores were found to be lower in patients with chronic pain. NRS scores were positively correlated with physical functioning domain and negatively correlated with bodily pain domain in painful patients.

Although chronic pain is often recognized as one of their major problems in SCI patients, studies designed to investigate its effects on QoL and mood are still lacking. It has been reported that SCI patients with high pain intensity (a score of $\leqslant 42$, derived from an item (bodily pain) from the SF-36 scale itself) were found to have significantly lower QoL than those with lower levels of pain intensity for all the SF-36 domains, other than physical functioning. ${ }^{2}$ In this study, bodily pain from the SF-36 was used for pain intensity. Others have also found that neuropathic pain amplified the decrease in QoL associated with SCI. ${ }^{1,17}$ However, all those studies lack a subgroup with chronic pain. In the current study, we specifically grouped the SCI patients as either having chronic pain or not. We found that pain significantly lowered only two SF-36 domains (bodily pain and social functioning). As the social functioning subscale evaluates the degree to which physical or emotional problems interfere with social activities, those patients with chronic pain had lower scores of social functioning subscale, indicating frequent interference with normal social activities. On the other hand, it is not surprising that patients with chronic pain had lower scores of bodily pain. Overall, as

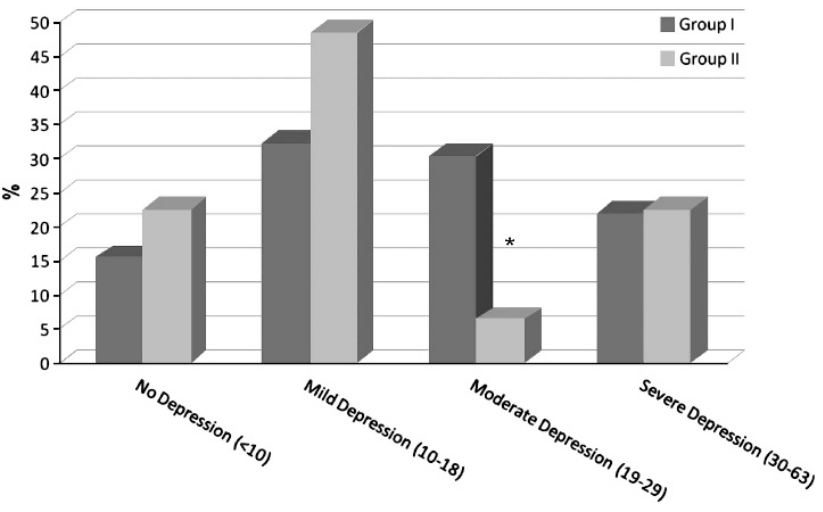

Figure 1 The comparison of Beck Depression Inventory scores between the groups according to the depression severity. ${ }^{*} P<0.05$.

Table 3 Mean scores of the SF-36 domains, BDI and motor-FIM

\begin{tabular}{llll}
\hline & Group I $(\mathrm{n}=109)$ & Group // $(\mathrm{n}=31)$ & $\mathrm{P}$ \\
\hline Physical functioning & $12.0 \pm 9.5$ & $10.3 \pm 5.2$ & 0.326 \\
Role_physical & $28.7 \pm 25.1$ & $21.8 \pm 17.9$ & 0.777 \\
Bodily pain & $46.7 \pm 19.4$ & $76.5 \pm 16.5$ & 0.000 \\
General health & $38.9 \pm 17.1$ & $39.8 \pm 17.1$ & 0.601 \\
Social functioning & $46.9 \pm 22.6$ & $56.4 \pm 23.5$ & 0.016 \\
Role-emotional & $28.9 \pm 25.3$ & $28.8 \pm 25.1$ & 0.532 \\
Vitality & $43.2 \pm 17.08$ & $47.6 \pm 16.1$ & 0.307 \\
Mental health & $49.9 \pm 18.02$ & $51.9 \pm 17.9$ & 0.943 \\
BDI scale & $22.1 \pm 11.7$ & $17.1 \pm 12.2$ & 0.021 \\
Motor FIM scale & $35.2 \pm 15.3$ & $35.4 \pm 16.0$ & 0.942 \\
\hline Abbrit & &
\end{tabular}

Abbreviations: BDI, Beck Depression Inventory; FIM, Functional Independence Measure. Bold values show statistical significance $(P<0.05)$,

the social disadvantages arising from impairment and limitations are observed as a major problem with SCI, ${ }^{1,18}$ management of chronic pain may improve these patients' QoL.

Chronic pain and depression are closely related and there is increasing evidence that depression often coexists with pain. ${ }^{19}$ Findings show that a higher proportion of patients with SCI have more depressed mood than healthy persons. ${ }^{10}$ Therefore, the association between depressed mood and pain suggests that the long-term emotional distress experienced by these individuals is significantly influenced by the presence of pain. ${ }^{19}$ However, previous studies were performed using a self-reported questionnaire with various scales about mood and/or they did not have a control group with chronic pain. ${ }^{10,19-21}$ In the current face-to-face interview study, we showed that a patient who had chronic pain was more likely to have higher levels of depression.

According to previous studies, demographic and medical variables were not associated with pain locations or ratings of pain intensity. ${ }^{4,22,23}$ In addition, SCI characteristics such as age at SCI, duration after SCI and completeness of injury were not related to pain ratings. ${ }^{8}$ It has been shown that incomplete spinal injuries were more likely to result in pain than complete injuries, whereas no significant association between the severity of pain and completeness of injury was found. ${ }^{4}$ Similarly, other than the completeness of injury and employment status, we could not find any significant demographic variable to be in association with pain intensity.

A previous study from Turkey reported that falls were found to be common (37.0\%) among SCI patients. ${ }^{24}$ In another study from 
Turkey, ${ }^{25}$ it was reported that the most common cause of SCI were falls $(42.6 \%)$ and motor vehicle crash $(38.5 \%)$, similarly as in the current study (35.0 and $34.2 \%$, respectively). In addition, we found that the frequency of SCI due to falls was significantly higher in the painful group. SCI from falls included falls from trees, from rooftops (owing to sleeping on rooftops in summer when it is hot) and from structural framework, all of which involved young adults.

In conclusion, to our best notice, this is the first study displaying chronic pain's negative impact on the mood and social functioning subscale of QoL in SCI patients. We suggest that chronic pain should always be treated in a multidisciplinary setting where pharmacological, physical and psychological therapies are combined to improve QoL. Further, considering that falls and motor vehicle accidents are the most common causes of traumatic SCI, we imply that the prevention strategies should be focused mainly on these types of traumas to decrease the frequency of SCI in relevant countries.

\section{DATA ARCHIVING}

There were no data to deposit.

\section{CONFLICT OF INTEREST}

The authors declare no conflict of interest.

1 Westgren N, Levi R. Quality of life and traumatic spinal injury. Arch Phys Med Rehabil 1998; 79: 1433-1439.

2 Middleton J, Tran Y, Craig A. Relationship between quality of life and self-efficacy in persons with spinal cord injuries. Arch Phys Med Rehabil 2007; 88: 1643-1648.

3 Ehde DM, Jensen MP, Engel JM, Turner JA, Hoffman AJ, Cardenas DD. Chronic pain secondary to disability: a review. Clin J Pain 2003; 19: 3-17.

4 Modirian E, Pirouzi P, Soroush M, Karbalaei-Esmaeili S, Shojaei H, Zamani H. Chronic pain after spinal cord injury: results of a long-term study. Pain Med 2010; 11: 10371043.

5 Ravenscroft A, Ahmed YS, Burnside IG. Chronic pain after SCI. A patient survey. Spinal Cord 2000; 38: 611-614.

6 Norrbrink BC, Hultling C, Lundeberg T. Quality of sleep in individuals with spinal cord injury: a comparison between patients with and without pain. Spinal Cord 2005; 43 $85-95$.
7 Westgren N, Hultling C, Levi R, Seiger A, Westgren M. Sexuality in women with traumatic spinal cord injury. Acta Obstet Gynecol Scand 1997; 76: 977-983.

8 Ullrich PM, Jensen MP, Loeser JD, Cardenas DD. Pain intensity, pain interference and characteristics of spinal cord injury. Spinal Cord 2008; 46: 451-455.

9 Craig AR, Hancock KM, Dickson HG. A longitudinal investigation into anxiety and depression in the first 2 years following a spinal cord injury. Paraplegia 1994; 32: 675-679.

10 Kennedy P, Rogers BA. Anxiety and depression after spinal cord injury: a longitudinal analysis. Arch Phys Med Rehabil 2000; 81: 932-937.

11 Rintala DH, Loubser PG, Castro J, Hart KA, Fuhrer MJ. Chronic pain in a communitybased sample of men with spinal cord injury: prevalence, severity, and relationship with impairment, disability, handicap, and subjective well-being. Arch Phys Med Rehabil 1998; 79: 604-614.

12 Beck AT, Steer RA. Internal consistencies of the original and revised Beck Depression Inventory. J Clin Psychol 1984; 40: 1365-1367.

13 Kendall PC, Hollon SD, Beck AT, Hammen CL, Ingram RE. Issues and recommendation regarding use of the Beck Depression Inventory. Cognit Ther Res 1987: 3: 289-299.

14 Siddall PJ, Taylor DA, Cousins MJ. Classification of pain following spinal cord injury. Spinal Cord 1997; 35: 69-75.

15 Hanley MA, Masedo A, Jensen MP, Cardenas D, Turner JA. Pain interference in persons with spinal cord injury: classification of mild, moderate, and severe pain. J Pain 2006; 7: 129-133.

16 Yücel A, Şenocak M. Results of the Leeds assessment of neuropathic symptoms and signs pain scale in Turkey: a validation study. Pain 2004; 5: 427-432.

17 Vall J, Batista-Braga VA, Almeida PC. Central neuropathic pain and its relation to the quality of life of a person with a traumatic spinal cord injury. Rev Neurol 2006; 42 525-529.

18 Kreuter M, Siosteen A, Erkholm B, Bystrom U, Brown DJ. Health and quality of life of persons with spinal cord lesion in Australia and Sweden. Spinal Cord 2005; 43: 123-129.

19 Budh CN, Osteraker AL. Life satisfaction in individuals with a spinal cord injury and pain. Clin Rehabil 2007; 21: 89-96.

20 Saikkonen J, Karppi P, Huusko TM, Dahlberg A, Mäkinen J, Uutela T. Life situation of spinal cord-injured persons in Central Finland. Spinal Cord 2004; 42: 459-465.

21 Cruz-Almeida Y, Alameda G. Widerström-Noga EG. Differentiation between pain-related interference and interference caused by the functional impairments of spinal cord injury. Spinal Cord 2009; 47: 390-395.

22 Demirel G, Yilmaz H, Gencosmanoğlu B, Kesiktaș N. Pain following spinal cord injury. Spinal Cord 1998; 36: 25-28.

23 Cardenas DD, Bryce TN, Shem K, Richards JS, Elhefni H. Gender and minority differences in the pain experiences of people with spinal cord injury. Arch Phys Med Rehabil 2004; 85: 1774-1781.

24 Karacan I, Koyuncu H, Pekel O, Sumbuloglu G, Kirnap M, Dursun H et al. Traumatic spinal cord injuries in Turkey: a nation-wide epidemiological study. Spinal Cord 2000, 38: 697-701.

25 Unsal-Delialioglu S, Kaya K, Sahin-Onat S, Kulakli F, Culha C, Ozel S. Fever during rehabilitation in patients with traumatic spinal cord injury: analysis of 392 cases from a national rehabilitation hospital in Turkey. J Spinal Cord Med 2010; 33: 243-248. 